Pathologe 2017 · 38:1-2

DOI 10.1007/s00292-017-0267-z

Online publiziert: 17. Januar 2017

๑) Springer-Verlag Berlin Heidelberg 2017

CrossMark

\section{K. W. Schmid}

Institut für Pathologie und Neuropathologie, Universitätsklinikum Essen, Essen, Deutschland

\title{
Die olympische Devise der Pathologie
}

Die Umfrageergebnisse des in diesem Heft erscheinenden Beitrags, „Was wünschen unsere Einsender. Ist Geschwindigkeit alles?", aus dem Institut für Pathologie des Universitätsklinikums Bonn [1] vermitteln durchaus den Eindruck, dass in Abwandlung der olympischen Devise "citius, altius, fortius“ [2] die offensichtliche Devise für unsere Tätigkeit „schneller, freundlicher, telefonisch erreichbarer" lauten muss. Auch wenn der Rücklauf mit nur 3,2 \% aller angeschriebenen Einsender tatsächlich die Frage der Repräsentativität der Umfrage stellt und möglicherweise noch die Antworten eher von Einsendern stammen, die der Wunsch nach Verbesserung des zum Zeitpunkt der Umfrage bestehenden Zustandes getrieben hat, befällt mich doch Verwunderung und Ärger; und ich will auch gar nicht verhehlen, dass der Ärger über eine meiner Ansicht nach doch sehr despektierliche und ignorante Betrachtung unserer Tätigkeit deutlich überwiegt.

Vorausgesetzt, dass kein Pathologe Fälle absichtlich „abliegen lässt“, bevor er deren Ergebnisse übermittelt, zeugt die konstante Forderung nach (noch) „schnellerer" Befundung in erster Linie von weitgehender Ahnungslosigkeit über den aufwendigen Prozess, der einem fertigen Befundbericht vorausgeht, obwohl im Wechselspiel von Labormaschinen, Computertechnik und Mensch heute schon fast jede Minute des 24-StundenTages (ggf. auch unter Einschluss von Wochenenden und Feiertagen) optimiert genutzt wird. Jeder Patient hat zweifelsfrei ein Anrecht, so schnell wie möglich seine Befunde zu erhalten. Wenn dies aber aufgrund mangelhafter Fixierung oder verkürzter Bearbeitungsschritte zur mangelhaften Qualität der Schnitte und Färbungen führt, sind die Grenzen der Beschleunigung unserer Befundtätigkeit längst überschritten; besonders bedenklich wird mangelhaft prozediertes Untersuchungsmaterial, wenn es molekularpathologische Analysen (z. B. beim Tumorprofiling) unmöglich oder nur eingeschränkt beurteilbar macht und dadurch für eine Therapieentscheidung elementare Veränderungen unentdeckt bleiben (müssen). Im täglichen Arbeitsablauf einer Pathologie kann vieles nur sequenziell und nicht parallel abgearbeitet werden; verantwortungsvoller Umgang mit den Kosten, insbesondere von Spezialuntersuchungen, kann auch zur Verlängerung der Entscheidungsfindung beitragen. Dazu sind etliche Fälle eben keine simplen „Blickdiagnosen“; für die Bearbeitung schwieriger Fälle braucht der Pathologe eben entsprechend Zeit, Literaturstudium, fachliche Diskussion und/oder externe konsiliarische Beratung. Manchmal sind auch (weitere) klinische Informationen für Befundentscheidungen essenziell und an diese muss man auch erst einmal kommen! In diesem Kontext erscheint es auch bezeichnend, dass in der Umfrage Parametern wie einem besser organisierten Probentransport, dem Einsatz von echten Eilschnitten (bei dezidierter klinischer Indikation) oder intraoperativen Gefrierschnitten deutlich weniger Gewicht als der generalisierenden Forderung „Befundgeschwindigkeit" beigemessen wird.

Was machen Pathologen und ihr Umfeld zwischenmenschlich so falsch, dass die "Freundlichkeit des Kontakts" in so hohem Maße eingefordert wird? Vielleicht bin ich betriebsblind, ich habe aber durchaus den Eindruck, dass Pathologen in der Regel sehr kultivierte und umgäng- liche Menschen sind. Dass wir gelegentlich in Tumorboards etc. unseren fachlich fundierten Standpunkt klar und deutlich machen müssen, kann uns wohl schwerlich negativ angerechnet werden. Und in den meisten Sekretariaten von pathologischen Instituten und Praxen sitzen echte Perlen, durch deren Charme, Langmut und organisatorisches Geschick vieles für unsere klinischen Partner viel einfacher gemacht wird.

Dies leitet auch direkt zur nächsten Forderung nach "telefonischer Erreichbarkeit" des Befunderstellers über; trotz aller technischen Unterstützung (Mobiltelefone, Freisprechanlagen, SMS, E-Mail etc.) kann es unsere Tätigkeit (z. B. Zuschnitt, Obduktion, klinischpathologische Konferenz, aber natürlich auch Lehre) mit sich bringen, nicht immer und überall erreichbar zu sein; vielleicht möchte man aber auch einmal bei der Bearbeitung eines anspruchsvollen Falles einfach nicht gestört werden. Die fachliche Fortbildung betreiben Pathologen masochistisch ohnehin so gut wie ausschließlich an zahllosen Wochenenden.

Aber vielleicht sollte man den Ärger über die durch die Umfrage der Bonner Universitätspathologie erzielte Prioritätenliste der Erwartungen der Einsender an Pathologen einfach hinunterschlucken und alles eher positiv sehen. Laut Umfrageergebnis scheint es mit der Qualität der Befunde wenig Probleme zu geben; sie sollten nur alle einen einheitlichen Befundaufbau mit gleichartiger Diktion der Befundformulierung, ggf. natürlich unter Einschluss von UICC (TNM) und Tumorverschlüsselung nach ICD, zeigen.

Was mich persönlich aber am positivsten stimmt, ist, dass ich ganz viele Klini- 
ker aus unterschiedlichsten Fachrichtungen kenne, die die verantwortungsvolle diagnostische Tätigkeit und den Einsatz ihrer Pathologen kennen, verstehen und sehr hoch schätzen und durchaus keine Scheu haben, dies auch Patienten gegenüber und auf Fachkongressen zum Ausdruck zu bringen.

In diesem Sinne verbleibe ich mit besten kollegialen Grüßen,

Ihr

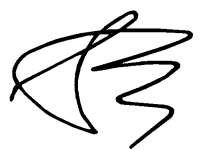

Prof. Dr. K. W. Schmid

Editor-in-Chief

\section{Korrespondenzadresse}

\section{Prof. Dr. K. W. Schmid}

Institut für Pathologie und Neuropathologie, Universitätsklinikum Essen

Hufelandstraße 55, 45122 Essen, Deutschland kw.schmid@uk-essen.de

Interessenkonflikt. K. W. Schmid gibt an, dass kein Interessenkonflikt besteht.

\section{Literatur}

1. Fronhoffs F, Thiesler F, Kristiansen G (2017) Was wünschen unsere Einsender. Ist Geschwindigkeit alles? Pathologe. doi:10.1007/s00292-015-0049-4

2. Müller N (2008) Die olympische Devise „citius, altius, fortius" und ihr Urheber Henri Didon. Abteilung Sportgeschichte Online Texte, Bd.11.

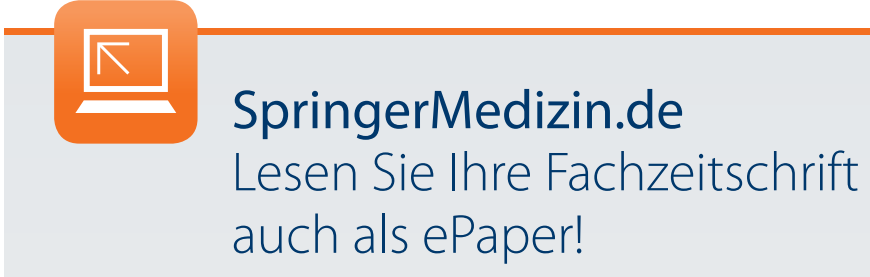

Als Abonnent können Sie Ihre Zeitschrift in verschiedenen Formaten lesen. Wählen Sie je nach Vorliebe und Situation aus, ob Sie die Zeitschrift als Print-Ausgabe, in Form von einzelnen Beiträgen auf springermedizin.de oder aber als komplette, elektronische ePaper-Ausgabe lesen möchten.

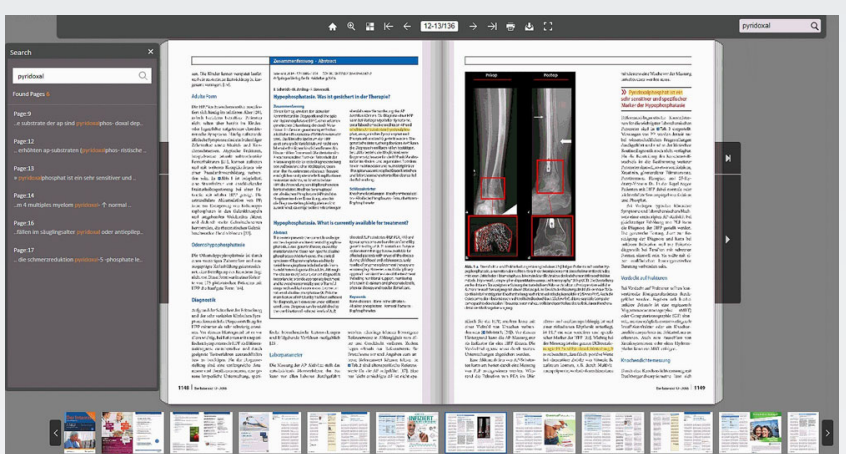

Die ePaper sind die identische Form der gedruckten Ausgaben. Sie sind nutzbar auf verschiedenen Endgeräten wie PC, Tablet oder Smartphone

Das sind die Vorteile des ePapers:

$>$ Das verlinkte Inhaltsverzeichnis führt Sie direkt zum gewünschten Beitrag.

> Eine Suchfunktion ermöglicht das Auffinden von Schlagworten innerhalb der Zeitschrift

$>$ Jede Ausgabe kann als PDF heruntergeladen und damit auch offline gelesen werden bzw. auch gespeichert oder ausgedruckt werden.

> Als Abonnent haben Sie Zugang zu allen ePaper-Ausgaben ab 2016.

Sie finden die ePaper auf SpringerMedizin.de bei der jeweiligen Ausgabe Ihrer Fachzeitschrift. Klicken Sie auf den Button „Zum ePaper". 